\title{
Myocutaneous flaps versus Fasciocutaneous flaps to cover exposed bone fractures of the leg (Comparative study)
}

\author{
A.A.Shoulah ${ }^{1}$, M.A.Khalil ${ }^{1}$, M.M.Nan ${ }^{2}$ and A.M.Abd El Mofeed ${ }^{1}$
}

${ }^{1}$ General Plastic Surgery, Dept.,Faculty of Medicine, Benha Univ., Benha, Egypt

${ }^{2}$ General Plastic Surgery, Dept., Benha Health Insurance Hospital., Benha, Egypt

E-Mail: marihannan13@gmail.com

\begin{abstract}
Open tibial cracks are serious wounds. The high energy regularly granted to the crack site upsets the delicate tissue envelope and strips the periosteum inclining to weakened recuperating and contamination. The target of this investigation is to look at the impacts of delicate tissue inclusion by one or the other muscle or fasciocutaneous tissues on the recuperating of open tibial cracks, This examination included 30 patients with post horrendous leg delicate tissue deserts from division of plastic medical procedure at Benha University Hospital and Benha Health Insurance Hospital, in Qaliubya, , in the time frame between December 2019 and December 2020, Patients' age went from 25 to 60 years of age. 26 cases were guys and 4 cases were females. 18 cases were related with comorbidities, while 12 cases were free. Altogether, 15 neighborhood muscle folds (half) and 15 nearby fasciocutaneous folds (half), were utilized for reproduction with (100\%) mending rate muscle folds, while the intricacy rate in the fasciocutaneous folds were $(20 \%)$. In the whole patients, 6 patients (20\%) built up a postoperative inconvenience, The mean length of emergency clinic stay in days was $9 \pm 3$ for the muscle fold gathering and $14 \pm 5$ for the fasciocutaneous fold gathering. The mean postpone time between the injury and the activity frequency in muscle fold bunch was $4(3-24)$ weeks and $4(1-8)$ in fasciocutaneous fold gathering. muscle ought to be the tissue of decision for covering high-energy Grade IIIB open distal tibial shaft cracks.
\end{abstract}

Keywords: Myocutaneous, flap, Fasciocutaneous, bone, fracture, leg.

\section{Introduction}

Open tibial cracks are extreme wounds. The high energy frequently granted to the break site disturbs the delicate tissue envelope and strips the periosteum inclining to debilitated recuperating and disease [1].

Recuperation can be delayed. with an opportunity to break association postponed contrasted with shut cracks. The generally uncovered subcutaneous situation of the tibia implies that it is especially inclined to such injury, where generous loss of overlying delicate tissues, periosteal stripping, and bone openness are portrayed as Gustilo IIIB wounds [2].

The job of delicate tissue reproduction in open cracks isn't restricted to twisted inclusion to forestall wound drying up and contamination. Delicate tissues additionally add to crack fix by filling in as a nearby wellspring of stem or osteoprogenitor cells, development factors, and vascular supply.[3]

The helpless vascularization and ensuing helpless mending experienced in this area frequently lead to delayed openness of bone or ligaments, bringing about disease or putrefaction [4].

The critical part of delicate tissue folds in lower appendage injury is to fill in as a wellspring of vascular stockpile to bone finishes that have been deprived of periosteum and gone through disturbance of the endosteum. [5]

The anteromedial surface of the leg has no strong bed and is covered with just skin and subcutaneous fat layer on the tibia. Besides, the skin and subcutaneous fat layer of anteromedial surface of the leg is more slender than different regions. Therefore, the delicate tissue of this territory is oftentimes harmed and whenever it is harmed, it effectively break down to a recalcitrant delicate tissue imperfection with openness of the cortex of the tibia [6].

Strategies for inclusion have advanced from basic unwinding calf entry point, neighborhood arbitrary folds and cross leg pedicle folds to nearby muscle folds, fasciocutaneous folds and microvascular free folds. The decision of fold in leg reproduction is dictated by the area of the imperfection, the size of the deformity, giver site dismalness, and the situation with the beneficiary vessel [7].

Break fix requires the enrollment of osteoprogenitor cells. Mesenchymal undifferentiated organisms are, by definition, multipotent, and can in this way fill in as a wellspring of osteoprogenitor cells.. In shut cracks, the principle wellsprings of osteoprogenitor cells are believed to be the bone marrow and periosteum. Notwithstanding, high-energy open breaks of long bones are described by loss of the periosteum and bone marrow, particularly following addition of an intramedullary pole. Under these conditions, the principle osteoprogenitor cells should begin from the neighborhood delicate tissues or the dissemination [8].

Muscle likewise gives a bone anabolic climate through the declaration of individuals from the changing development factor- $\beta$ superfamily of development and separation factors, including the bone morphogenetic proteins. The equal connection among muscle and bone mass is very much portrayed, especially the solid relationship between sarcopenia (age-related loss of bulk) and osteopenia. Muscle and bone are accepted to be commonly directing by methods for actual powers and cytokine control. Undoubtedly, ongoing proof demonstrates that muscle fills in as an endocrine organ that discharges trophic variables, known as myokines, which have been recognized as key controllers of the muscle and bone mass. Further perceptions recommend that unblemished muscle underpins bone fix by methods for the arrival of bone anabolics, including insulin-like development factor-1, interleukin-6, mind inferred neurotrophic factor, and fibroblast development factor-2 [9] 
Though seriously harmed muscle, debilitates this cycle through the arrival of catabolic myokines, including myostatin (development differentiationfactor-8).

Subsequently, the net impact on bone is subject to the equilibrium of these components [10].

The goal of this investigation is to think about the impacts of delicate tissue inclusion by one or the other muscle or fasciocutaneous tissues on the mending of open tibial breaks.

\section{Patients and methods}

This examination included 30 patients with post horrible leg delicate tissue deserts from division of plastic medical procedure at Benha University Hospital and Benha Insurance Hospital, in Qaliubya, in the time frame between December 2019 and December 2020, Patients' age went from 25 to 60 years of age. 26 cases were guys and 4 cases were females.

A composed educated assent clarifying the entire system under investigation in this examination including photography has been acquired from all patients and total classification, as respect the patients' names and addresses, was given uncommon consideration as per nearby moral board of trustees of Benha Faculty of Medicine.

All cases were related with open tibial breaks (Gustilo grades IIIb); 15 cases were treated by muscle folds and 15 were treated by fasciocutanious folds. The imperfections differed in size between $3 \mathrm{~cm} \mathrm{x3} \mathrm{cm}$ to 14 $\mathrm{cm} \times 10 \mathrm{~cm} .27$ cases were related with comorbidities (Hypertension, 18 cases; Smoker 9 cases and 9 cases were diabetic, while 3 cases were free.

\subsection{Inclusion criteria}

- Patients complaining of post traumatic exposed tibial fractures.

- Both gender were included.

\subsection{Exclusion criteria}

- Patients under the age of 15 years

- Patients over the age of 65 years.

- Patients with ischemic diseases.

- Patients with extensive vascular injury.

The study included two groups as follows:

- Group I: Fifteen cases with muscle flap.

- Group II: Fifteen cases with fasciocutaneous flap.

All patients were subjected to full history taking, complete clinical examination, and Investigations as: Full laboratory investigations, Radiological investigations: XRay to detect bony fractures, Duplex to assess the vascular condition of the limb, CT angiography if free tissue transfer was planed.

\subsection{Statistical methods}

Information the board and factual examination were finished utilizing SPSS vs.25. (IBM, Armonk, New York ,United States). Mathematical information were summed up as means and standard deviations or medians and reaches. Straight out information were summed up as numbers and rates. Examinations between the two gatherings were finished utilizing autonomous $t$ test or Mann Whitney U test for typically and non-regularly conveyed mathematical information, separately. Straight out information were looked at utilizing the Chi-square test or Fisher's definite test if fitting. All P esteems were two-sided. $\mathrm{P}$ esteems under 0.05 were viewed as huge.

\section{Results}

There were no significant differences between both groups as regard gender and age, 26 of them were males $(86.6 \%), 4$ were females $(13.3 \%)$. The mean age incidence of the patients was $52 \pm 14 \& 47 \pm 6$ ranged from $25-60$ years ,P values were 0.598 and 0.25 , respectively Table (1)

Table (1) Demographic characteristics of both groups.

\begin{tabular}{llccc}
\hline & & Muscle flap $(\mathbf{n}=\mathbf{1 5})$ & Fascio-cutaneous flap $(\mathbf{n}=\mathbf{1 5})$ & P value \\
\hline Age (years) & Mean $\mathbf{4 S D}$ & $52 \pm 14$ & $47 \pm 6$ & 0.25 \\
Gender & Males n (\%) & $12(80.0)$ & $14(93.3)$ & 0.598 \\
& Females n (\%) & $3(20.0)$ & $1(6.7)$ & \\
\hline
\end{tabular}

Chi-square or Fisher's exact test was used.

According to comorbidity, 12 cases were not having any concomitant disease (40\%) while 18 cases were associated with comorbidities $(60 \%)$; 9 cases were smokers (30\%), 12 cases were hypertensive (40\%) and 6 cases were diabetics (20\%) fig (1).

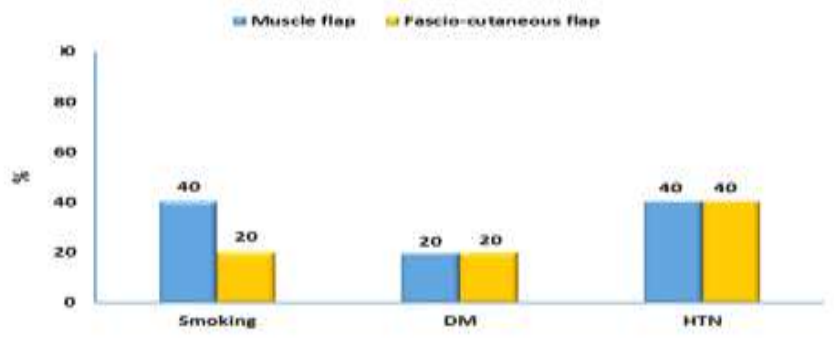

Fig. (1) Risk factors distribution in both groups 
There were no significant differences between both groups as regard delay time between the trauma and the operation.
The mean delay time incidence in Group I was 4 (3 24) weeks \& $4(1-8)$ in Group II ,P values was 0.267 fig(2).

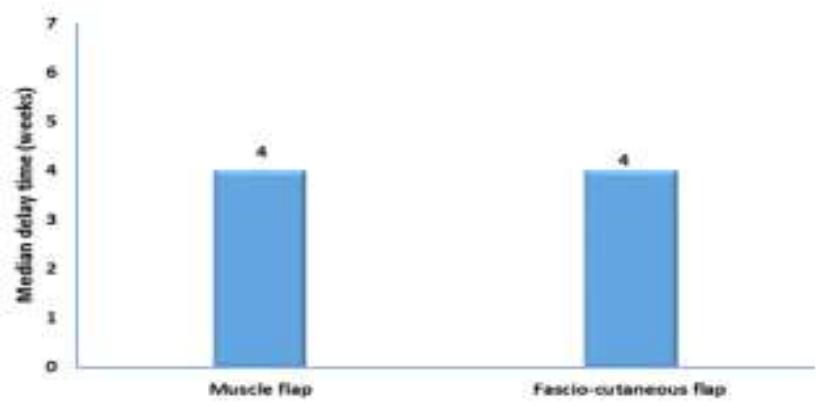

Fig. (2) Delay time in both groups.

Flap types in Fascio-cutaneous flap group.

Fascio-cutaneous flap types included Pedicled Posterior tibial perforator $(20.0 \%)$, Bipedicled $(20.0 \%)$,
Cross leg (20.0\%), Local transposition (20.0\%), Propellar $(20.0 \%)$ fig (3).

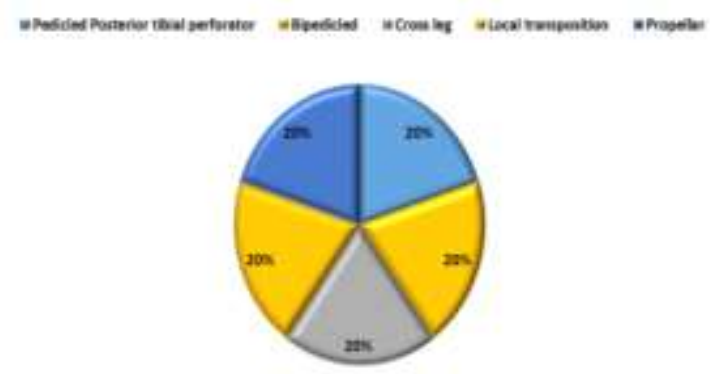

Fig. (3) Flap types in fascio-cutaneous flap group.

Flap types in muscle flap group.

Muscle flap types included Gastrocnemius and splitthickness skin graft $(40.0 \%)$, Hemi soleus and split- thickness skin graft $(40.0 \%)$, and Tibialis anterior and split-thickness skin graft $(20.0 \%)$ fig (4).

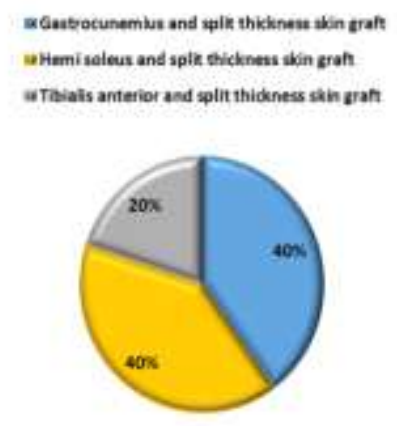

Fig. (4) Flap types in muscle flap group.

In the Group I $(n=15)$ there were 15 patients $(100 \%)$ show complete flap survival while in Group II $(n=15)$ there was 12 patients $(80 \%)$ show complete flap survival. In Group II $(n=15) 3$ patients $(20 \%)$ show partial flap necrosis, after which the necrotic part of the flap was debrided and skin grafted in 1 case (6.6), left to heal by secondary intention in 1 case (6.6), re-advancement of the flap in 1 case (6.6). Complete flap survival showed no statistically significant difference between both groups. Pvalue was 0.224 .

In the Group I $(n=15)$ there were 14 patients $(93.3 \%)$ show no recipient site infection while in Group II $(n=15)$ 
there was 12 patients $(80 \%)$ show no recipient site infection. In Group I $(n=15) 1$ patient $(6.7 \%)$ show recipient site infection and in Group II $(n=15) 3$ patients (20\%) show recipient site infection, all patients were treated conservatively by antibiotics according to culture and sensitivity test and frequent dressing until healing. Postoperative recipient site infection showed no statistically significant difference between both groups. Pvalue was 0.598 .

After 3 months we checked the primary bone healing, a direct attempt by the cortex to establish itself without formation of a fracture callus, in the Group I $(n=15)$ there were 14 patients $(93.3 \%)$ show primary bone healing while in Group II $(n=15)$ there was 12 patients $(80 \%)$ show primary bone healing. In Group I $(n=15) 1$ patient $(6.7 \%)$ show delayed primary bone healing and in Group II $(n=15) 3$ patients (20\%) no primary bone healing. Primary bone healing showed no statistically significant difference between both groups. P-value was 0.598 Table (2).

Table (2) Primary bone healing in both groups.

\begin{tabular}{lllccc}
\hline & & & Muscle flap $(\mathbf{n}=\mathbf{1 5})$ & Fascio-cutaneous flap $(\mathbf{n}=\mathbf{1 5})$ & P value \\
\hline Primary bone healing & Good & $\mathbf{n}(\boldsymbol{\%})$ & $14(93.3)$ & $12(80.0)$ & 0.598 \\
& Fair & $\mathbf{n}(\boldsymbol{\%})$ & $1(6.7)$ & $3(20.0)$ & \\
\hline
\end{tabular}

Fisher's exact test was used.

After 9 months we checked the overall bone healing in Group I $(n=15)$ there were 15 patients $(100 \%)$ show bone healing while in Group II $(n=15)$ there was 12 patients $(80 \%)$ show bone healing.
In Group II $(\mathrm{n}=15) 3$ patients $(20 \%)$ nonunion and were referred to orthopedic department for further assessment. The bone nonunion showed no statistically significant difference between both groups. P-value was 0.224 Table (3).

Table 3: Bone nonunion in both groups.

\begin{tabular}{lcccc}
\hline & & Muscle flap $(\mathbf{n}=\mathbf{1 5})$ & Fascio-cutaneous flap $(\mathbf{n}=\mathbf{1 5})$ & P value \\
\hline Bone nonunion & N\% & $0(0.0)$ & $3(20.0)$ & 0.224 \\
\hline
\end{tabular}

Fisher's exact test was used

No donor site morbidity was reported in both groups. The mean length of hospital stay in days was $9 \pm 3$ for the the muscle flap group and $14 \pm 5$ for the fasciocutaneous flap group Table (4).
The post-operative hospital stay was significantly higher in the fascio-cutaneous flap group (14 days) than the muscle flap group ( 9 days). P-value was 0.013

Table (4) Post-operative hospital stay in both groups.

\begin{tabular}{lcccc}
\hline & & Muscle flap $(\mathbf{n}=\mathbf{1 5})$ & Fascio-cutaneous flap $(\mathbf{n}=15)$ & P value \\
\hline Post-op. hospital stay (days) & Mean \pm SD & $9 \pm 3$ & $14 \pm 5$ & 0.013 \\
\hline
\end{tabular}

\subsection{The Independent t-test was used}

Patients were monitored for post-operative complications which divided into; early recipient site complications such as: infection, wound dehiscence and flap necrosis; late recipient site complications such as: disability, recurrence, deformity, delayed bone healing and scars ; donor site morbidity. In the entire patients $(n=30), 6$ patients $(20 \%)$ developed a postoperative complication, 3patient in Group I $(n=15)$, consisted of postoperative recipient site infection and fair primary bone healing in one patient (6.6) and muscle bulkiness in two patients (13.3). 3 patient in Group II $(n=15)$, which consisted of recipient site infection, delayed primary bone healing, nonunion of the fracture, and development a partial flap necrosis. 24 cases $(80 \%)$ showed satisfactory results in form of complete healing and coverage of the defect, no recipient site infection, good primary bone healing, union of the fracture, no donor site morbidity and early restoration of function.

There was no significant difference between both groups as regard complications. P-value was 1.0 as we used small sample of patients to monitoring but overall bone healing was better in patients treated $b$ muscle group.

\section{Discussion}

In the whole examination post-employable difficulties created in 6 patients $(20 \%)$ as halfway fold corruption in the four cases.

This post-employable difficulty rate is in rough match with the inconvenience rate in the investigation by Franken [11] who created (31\%) entanglement rate.

It additionally match what Hallock GG [12] found in his examination as he evaluated the part of muscle and 
sash folds in lower furthest point injury. Subtleties of fold inclusion in 160 appendages in 155 patients, of which 60 were nearby muscle, 50 were neighborhood fascial, and 74 were free muscle and fascial folds, were accounted for. Difficulties were identified with the seriousness of the injury, with $39 \%$ related with free fold move, while neighborhood muscle and nearby belt folds had comparable morbidities of $27 \%$ and $30 \%$, individually.

Early test examines including murine and canine models exhibited improved callus development and higher bone mineral thickness under muscle folds and prevalent end of organisms in the injury bed contrasted and fasciocutaneous folds. Moreover, mesenchymal foundational microorganisms from muscle show a more noteworthy potential for osteogenesis. [13]

In this examination, neighborhood muscle folds were utilized effectively in 15 cases (half of cases) at that point covered with split thickness skin unite as Gastrocnemius in 6 cases $(40 \%)$, Hemi soleus in 6 cases $(40 \%)$ and Tibialis foremost in 3 cases $(20 \%)$

We utilized fasciocutaneous folds to cover deserts in 15 patients (half of cases) as Pedicled back tibial perforator in 3 cases $(20 \%)$, Bipedicled fold in 3 cases (20\%), Cross leg fold in 3 cases (20\%), Local interpretation fold in 3 cases $(20 \%)$ and Propeller fold in 3 cases $(20 \%)$

Complete fold endurance in muscle bunch was $100 \%$ ( 15 of 15 fold) while in fasciocutaneous folds was $80 \%$ (12 of 15 fold) as three folds were confounded hy halfway fold rot, after which the necrotic piece of the fold was debrided and skin united in 1 case (6.6), left to mend by auxiliary goal in 1 case (6.6), re-progression of the fold in 1 case (6.6)

Postoperative beneficiary site disease in muscle bunch was $6.7 \%$ ( 1 of 15 fold) while in fasciocutaneous folds was folds was $20 \%$ (3 of 15 fold) that were oversee moderately with anti-toxin and wound consideration

There is phenomenal coordinating between our investigation and that of Georgiadis [14] featured the capacity of muscle folds to lessen both mending time and profound disease. while Small and Mollan [15] reflectively investigated 168 open tibial breaks treated over a 15-year time frame and found a lower pace of putrefaction in nearby muscle folds (13.3 percent) contrasted and fasciocutaneous folds (21.2 percent)

The pace of essential bone association in muscle bunch was $93.3 \%$ (14 of 15 fold) while in fasciocutaneous folds was folds was $80 \%$ (12 of 15 fold)

Bone nonunion that weakened weight bearing in muscle bunch was $0 \%$ (0 of 15 fold) while in fasciocutaneous folds was $20 \%$ (3 of 15 fold). used to assess break mending, was doled out to patients' radiographs postoperatively, at 3, 6, and a year from the underlying crack date.

Mehta and Abdou [16] contemplated A radiographic association score for tibia (RUST) cracks that show a huge distinction $(\mathrm{P}=0.026)$ in the mean RUST score at a half year between the muscle gathering (8.54 61.81$)$ and the fasciocutaneous gathering $(6.926$ 2.46). There was no huge distinction in the mean RUST score at 3 months $(\mathrm{P}=$
0.056) and at a year $(\mathrm{P}=0.947)$ between the 2 gatherings. There was additionally importance in the quantity of breaks arriving at association, preferring muscle folds, at a half year $(\mathrm{P}=0.020)$

The examination led by us and Mehta\& Abdou [16] showed that patients with tibial breaks who got muscle folds have fundamentally quicker radiographic movement of bone recuperating in the initial a half year than do patients who got fasciocutaneous folds. Besides, tibia breaks getting muscle folds arrive at association by a half year than those fluttered with fasciocutaneous tissue.

Interestingly with our investigation Harry [17] exhibited higher vessel thickness in mouse tibia breaks reproduced with fasciocutaneous tissue during all phases of crack recuperating contrasted and muscle. The predominance of muscle folds regarding bone mending and paces of disease demonstrated by these early investigations including creature models, be that as it may, has not meant prevalent clinical results and Paro [18] figured out how to weight bearing in patients recreated with fasciocutaneous folds, albeit these patients were bound to require debulking later on.

In our examination we found no benefactor site grimness occurred in the two gatherings, Although Hallock [19] looked at the relative giver site bleakness of muscle and fascial folds. Altogether, 147 neighborhood muscle/musculocutaneous and 122 sash/fasciocutaneous folds were utilized to remake all districts of the body. These incorporated a sum of 45 muscle and 72 fasciocutaneous folds for the lower appendage, in spite of the fact that it was not satisfactory whether every one of these patients had uncovered breaks. Generally speaking, contributor site confusions were comparable at $14 \%$ for each gathering. Most challenges, be that as it may, were experienced underneath the knee with fasciocutaneous fold benefactor destinations, where no neighborhood muscle alternative was accessible, and the skin-joined giver locales were depicted as cosmetically unappealing.

\section{Conclusion}

We have portrayed a novel in patients of Grade IIIB open tibial breaks that has empowered the investigation of the elite impacts of muscle and fasciocutaneous tissue, and endeavors to go some path in concluding which ought to be the tissue of decision when planning folds for crack inclusion. Our information proposes that muscle is unrivaled in advancing the mending of breaks deprived of periosteum. Accorgingly, muscle ought to be the tissue of decision for covering high-energy Grade IIIB open distal tibial shaft breaks.

\section{References}

[1] R.B. Gustilo, R. L. Merkow, and D. Templeman, "The management of open fractures.," JBJS, vol. 72, pp. 299-304, 1990.

[2] S. Gopal, P. V Giannoudis, A. Murray, S. J. Matthews, and R. M. Smith, "The functional outcome of severe, open tibial fractures managed with early fixation and flap coverage," J. Bone Joint Surg. Br., vol. 86, pp. 861-867, 2004. 
[3] R. Liu, A. Schindeler, and D. G. Little, "The potential role of muscle in bone repair," J Musculoskelet Neuronal Interact, vol. 10, pp. 71-76, 2010.

[4] L. Hollier, S. Sharma, E. Babigumira, and M. Klebuc, "Versatility of the sural fasciocutaneous flap in the coverage of lower extremity wounds.," Plast. Reconstr. Surg., vol. 110, pp. 1673-1679, 2002.

[5] R. R. Richards and E. H. Schemitsch, "Effect of muscle flap coverage on bone blood flow following devascularization of a segment of tibia: an experimental investigation in the dog," J. Orthop. Res., vol. 7, pp. 550-558, 1989.

[6] T. M. N. Ata-ul-Haq, F. S. Malik, K. Khalid, A. Riaz, M. Y. Mehrose, and H. Khan, "Hemisoleus muscle flap, a better option for coverage of open fractures involving middle third of tibia," J Ayub Med Coll Abbottabad, vol.21, pp. 154-158, 2009.

[7] S. S. Gad, T. F. Kishk, S. M. Elkashty, and M. K. Bassam, "Reconstruction of the middle third of the leg by distally based hemigastrocnemius muscle flap,” Menoufia Med. J., vol. 31, pp. 1369, 2018.

[8] A. Schindeler, R. Liu, and D. G. Little, "The contribution of different cell lineages to bone repair: exploring a role for muscle stem cells," Differentiation, vol. 77, no. 1, pp. 12-18, 2009.

[9] B. K. Pedersen, "Muscles and their myokines," J. Exp. Biol., vol. 214, pp. 337-346, 2011.

[10] M. W. Hamrick, "A role for myokines in musclebone interactions," Exerc. Sport Sci. Rev., vol. 39,pp. 43, 2011.

[11] J. M. Franken, P. Hupkens, and P. H. M. Spauwen, "The treatment of soft-tissue defects of the lower leg after a traumatic open tibial fracture," Eur. J. Plast. Surg., vol. 33, pp. 129-133, 2010.
[12] G. G. Hallock, "Utility of both muscle and fascia flaps in severe lower extremity trauma," J. Trauma Acute Care Surg., vol. 48, pp. 913-917, 2000.

[13] J. K. K. Chan, L. Harry, G. Williams, and J. Nanchahal, "Soft tissue reconstruction of open fractures of the lower limb: muscle versus fasciocutaneous flaps," Plast. Reconstr. Surg., vol. 130 , pp. 284e, 2012.

[14] G. M. Georgiadis, F. F. Behrens, M. J. Joyce, A. S. Earle, and A. L. Simmons, "Open tibial fractures with severe soft-tissue loss. Limb salvage compared with below-the-knee amputation.," J. Bone Joint Surg. Am., vol. 75, pp. 1431-1441, 1993.

[15] J. O. Small and R. A. B. Mollan, "Management of the soft tissues in open tibial fractures," Br. J. Plast. Surg., vol. 45, pp. 571-577, 1992.

[16] D. Mehta et al., "Comparing radiographic progression of bone healing in Gustilo IIIB open tibia fractures treated with muscle versus fasciocutaneous flaps," J. Orthop. Trauma, vol. 32, pp. 381-385, 2018.

[17] L. E. Harry, A. Sandison, M. F. Pearse, E. M. Paleolog, and J. Nanchahal, "Comparison of the vascularity of fasciocutaneous tissue and muscle for coverage of open tibial fractures," Plast. Reconstr. Surg., vol. 124, pp. 1211-1219, 2009.

[18] J. Paro, G. Chiou, and S. K. Sen, "Comparing muscle and fasciocutaneous free flaps in lower extremity reconstruction-does it matter?," Ann. Plast. Surg., vol. 76, pp. S213-S215, 2016.

[19] G. G. Hallock, "Relative donor-site morbidity of muscle and fascial flaps.," Plast. Reconstr. Surg., vol. 92, pp. 70-76, 1993. 